Apuntes Universitarios, 2020: 10(3), julio-setiembre

ISSN: 2304-0335 DOI: https://doi.org/10.17162/au.v10i3.463

\title{
EI liderazgo: una visión desde las teorías organizacionales
}

\author{
Leadership: a view from organizational theories \\ María Olivia Palafox Soto, ${ }^{1 a}$ Sergio Ochoa Jiménez ${ }^{2}$ y Carlos Armando Jacobo- \\ Hernández ${ }^{3}$ \\ Instituto Tecnológico de Sonora, Ciudad Obregón, Sonora; México ${ }^{123}$ \\ (iD) https://orcid.org/0000-0002-8245-30321 \\ iD https://orcid.org/0000-0003-1848-37602 \\ (iD) https://orcid.org/0000-0002-8524-6258 3
}

Recibido: 12 de enero de 2020

Aceptado: 29 de abril de 2020

\section{Resumen}

El liderazgo es parte esencial para la gestión de las organizaciones, el cual ha sido estudiado desde diversas vertientes. Por ello, el presente artículo tiene por objetivo mostrar el abordaje teórico de dicho aspecto desde la visión de las teorías organizacionales, con un análisis histórico, analítico y crítico de las aportaciones, tomando como referencia la metodología propuesta por Fink, mediante una revisión de literatura. Lo anterior permite afirmar que la existencia del liderazgo en las organizaciones es dependiente de la coexistencia de motivación, comunicación, poder, autoridad, entre otros elementos, que le dan vida, presencia y esencia a todo tipo de organizaciones, contribuyendo así a la permanencia de las mismas.

Palabras clave: Liderazgo; gestión organizacional; teorías organizacionales; organización; poder.

\begin{abstract}
Leadership is an essential part of the organizational management, which has been studied from different perspectives. Therefore, this article shows the theoretical approach of these aspects from the perspective of organizational theories, with a historical, analytical and critical analysis of the contributions, taking as reference the methodology proposed by Fink, through a literature review. The above, allows to affirm that the existence of leadership in the organizations, is dependent on the coexistence of motivation, communication, power, authority, among other elements, that give it life, presence and essence in all types of organizations thus contributing to the permanence of the same.
\end{abstract}

Keywords: Leadership; Organizational Management; Organizational Theories; Organization; Power.

${ }^{a}$ Correspondencia al autor:

E-mail: sochoa@itson.edu.mx 


\section{Introducción}

El liderazgo es considerado un aspecto que ha logrado avances significativos en las organizaciones, permitiendo un cambio dentro de éstas, logrando afrontar nuevos retos ante la complejidad e incertidumbre del entorno (Gil et al., 2011). Dicho término refleja un esfuerzo y significado para la sociedad considerándose como la capacidad que tiene una persona para lograr influir en una o en un grupo de personas, ganando motivación y el dinamismo en ellas (Carrión, 2007).

El origen y evolución de liderazgo data del quehacer de los líderes, personas que centraron sus esfuerzos para lograr una mejor administración de países, aquellos que supieron dirigir a otros hombres, y de quienes planearon y organizaron guerras en las civilizaciones antiguas (Estrada, 2007a); es por ello la importancia que desde tiempo atrás mantiene el liderazgo en el logro de la eficiencia.

En las organizaciones, el liderazgo busca que los trabajadores se desarrollen en el largo plazo, estimulando intereses, habilidades y enfatizando la motivación con el fin de conseguir un objetivo mediante el direccionamiento de los mismos; poder comprender este aspecto proviene de lo social y humano por lo que se intenta cuidar tanto las necesidades como los intereses (Prentice, 2004). De acuerdo con Palomo (2013) el líder es una persona que desempeña distintos roles dentro de las organizaciones, funge de modelo para los trabajadores en el cumplimento de objetivos, es de gran ayuda fomentando el trabajo en equipo, la disciplina, el compromiso, entre otros aspectos.

Además, el liderazgo es considerado una aspiración en donde los individuos deben esforzarse diariamente por ser mejores, honestos, a proyectar una gran visión al mediano y largo plazo, inspirar a los demás y ser una persona constante, disciplinada y persistente en lograr lo que se propone (Posner \& Kouzes, 2011). Al mismo tiempo, querer tener un liderazgo efectivo en la organización es mediante el desarrollo de actividades, la presencia de una cultura de aprendizaje y también de la experiencia (Yukl, 2003).

De igual forma, el poder realizar esta investigación haciendo un recorrido desde los orígenes de la administración hace posible comprender el predominio y los aportes que ha tenido dicho aspecto dentro de las organizaciones donde han sido caracterizadas por conservar un estilo de liderazgo que las ayude a tener un mejor desempeño y funcionamiento (Estrada, 2007b). Asimismo, y habiendo establecido la importancia del liderazgo y lo necesario de ejercerlo dentro de las mismas, el objetivo del presente artículo es conocer el abordaje de las teorías organizacionales a través de una revisión de literatura que permita identificar diversos elementos que determinen la esencia del liderazgo 


\section{Revisión bibliográfica}

Con el estudio de las teorías organizacionales es posible encontrar infinidad de aspectos y hechos, que han evidenciado la forma en cómo trabajan las organizaciones; por lo cual, es posible encontrar ciertos elementos, donde se puede mostrar la forma en que gestionaban a las mismas, la manera de colaborar, mandar y controlar, con la finalidad de lograr el cumplimiento de objetivos y con el trabajo propuesto, por lo que en la presente investigación se analizarán aspectos que evidencian el uso de dicho aspecto.

\section{Teoría de la administración científica}

Comenzando con la administración científica, donde se dio inicio a la eficiencia industrial con Frederick Winslow Taylor, un ingeniero y economista conocido por ser una persona que se preocupaba en hacer las cosas bien y que otros hicieran lo mismo, consideraba que debían existir reglas equitativas que todos debían seguir, ofreciendo un trato justo, creía que los trabajadores debían ser bien remunerados, dándoles la oportunidad de ascender y percibir mejores ganancias (Taylor, 1983).

Taylor, aprendió el valor de esmerarse por la realización del trabajo y conseguir lo que fuese necesario por salir adelante, a partir de allí, se enfocó en lograr la máxima prosperidad y una mejor productividad, a través de una mejor administración, colocando a la persona correcta en los puestos altos, buscando así que el control y poder no esté concentrado en un solo individuo, sino en varias personas capaces de tomar decisiones importantes acerca de cualquier problema que se presente (Taylor, 1983).

Cabe mencionar, que la administración científica buscaba lograr su propósito mediante un Thinking Department y la súper especialización de los obreros de una forma estricta, suprimir el bajo rendimiento, para lo cual era necesario eliminar las mentiras, los malos sistemas así como los métodos ineficientes (Taylor, 1983); sin embargo, la idea de establecer este departamento, era con la finalidad de implantar nuevos jefes, apropiándose del pensamiento de los obreros y excluyéndolos de participación o iniciativa, todo lo que Taylor buscaba era un método rápido de querer hacer un intento por el crecimiento de la organización haciendo creer a los obreros trabajar bajo el lema de: "la mejor forma de hacer las cosas" (Ibarra y Montaño, 1992). 


\section{Teoría de la administración industrial}

Por otra parte, continuando con la revolución industrial y los aportes de Henri Fayol, un ingeniero que durante su trayectoria profesional creía necesario que el personal de las organizaciones, especialmente las grandes, requerían mayor organización; decidió involucrarse completamente para estudiar y analizar este aspecto, Henri llegó a crear una doctrina considerada como un aspecto fundamental en el campo administrativo, la cual, era conocida como fayolismo (Arévalo 2011).

Lo que propuso Fayol fue considerado un éxito, de manera que fue implementada por otros autores, consistía en facilitar a las organizaciones de cualquier tipo o sector, reglas, principios y políticas que las ayudasen a resolver cualquier problema o necesidad que se presentara, pues la administración es una función esencial que permite tener más capacidad en toda clase de empresas, si se ejecuta correctamente a través de los niveles jerárquicos y desarrollando ciertas cualidades y capacidades que le aportan a la organización un valor extra, tanto para los trabajadores como para los altos mandos de la misma (Fayol, 1987).

No obstante, de acuerdo con García y Dutchke (2007) afirman que Fayol quería esconder el poder absoluto mediante el rendimiento de la organización a través del trabajo especializado, implementando y reorganizando los procesos organizacionales en las tareas administrativas, enfocándose en los altos directivos y olvidándose de los puestos inferiores, dejando de lado que todo el recurso humano y el conocimiento se consideren valiosos en ámbitos profesionales, personales y/o sociales.

\section{Teoría de las relaciones humanas}

Del mismo modo, dentro de las teorías administrativas a través del tiempo se hace mención en la Teoría de las relaciones humanas (TRH) con su principal precursor Elton Mayo, un psicólogo preocupado por aspectos sociales, motivacionales, de productividad e ideología, tomándole importancia a la capacidad de las personas en distintos ámbitos dentro y fuera de la organización, considerándola como la sociología industrial (Mayo, 1946), por lo que realizó diversos experime ntos que ayudaron a conocer y entender la mejor forma de trabajar con el pensamiento humano.

Además, Mayo (1946), pensaba que las organizaciones se iban al fracaso no por la producción sino por las malas relaciones humanas que existían dentro de la misma; es por ello que en esta teoría era evidente el hecho que para establecer un buen liderazgo era mediante el orden y control dentro del ambiente de trabajo, a través de la observación y el trato hacia los demás. A pesar de que la TRH se inició manejando diversos aspectos que según traerían beneficios a las organizaciones tales como la 
motivación a los obreros, liderazgo a los directivos, una mejor comunicación, excelente dinámica y el hecho de estar basada en diversos principios para su funcionamiento como la integración social, recompensas o sanciones, aspectos emocionales, entre otros, ha tenido diversas críticas desde su surgimiento pues algunos autores exponen que a Mayo le falta validez científica, y tiene un alto grado de preocupación por la felicidad con una visión corta dando paso a producir diversos conflictos (Da silva, 2007).

Así pues, Mayo pensaba al igual que otros críticos, que el trabajo en una industria era monótono y fastidioso, por lo cual se teorizó en la disminución del mismo; sin embargo, según Gillespie (1991) menciona que cualquier trabajo lo era, ya que cada obrero se dedicaba a realizar las mismas actividades todo el tiempo por lo que interfería con la baja producción y rotación dentro de la organización, aunque esto no sea verídicamente experimentable.

\section{Teoría de la burocracia}

Continuando con aspectos sociales, un precursor interesado en ello fue Max Weber con la Teoría burocrática (TB), un economista y político quien tenía gran interés en estudiar a la sociedad humana, su funcionamiento, leyes, interrelaciones, comunicación, estructura, procesos y todo lo que influye en ella, además de enfocarse en temas como religión, familia, trabajo, política, entre otros con la finalidad de establecer un tipo ideal de organización (Weber, 1973); lo que se expone dentro de la TB es un liderazgo con base en dominación y autoridad a través del poder en la estructura de las organizaciones mediante ciertas reglas.

Por otro lado, de acuerdo con Carreño (2016) la burocracia deber estar entendida en todo su aspecto para que pueda enfrentar la economía con el poder y lograr la consecución de los intereses; además, según Assalone (2012) afirma que con ella se pueden reducir los conflictos sociales que se originan entre el gobierno del estado y la sociedad en general, ya que se encuentra dentro del ámbito político. Del mismo modo, Weber considera que la dominación es uno de los elementos que se deben considerar en las acciones de la comunidad, pues determina la finalidad de lo que se pretende lograr y permite el direccionamiento hacia metas establecidas (Weber, 1992). Precisamente lo anterior, ha generado fuertes críticas a los precursores, que buscan un futuro prometedor para las organizaciones (Mouzelis, 1975); ya que al implementarla se pensaba obtener más armonía, cuando en realidad solo se buscaba imponer el poder y la dominación en la toma de decisiones (Crozier, 1974). 


\section{Teoría del comportamiento}

También se encuentra la Teoría del comportamiento (TC) de Herbert Simon, en donde la administración plasma una referencia al arte de hacer las cosas de la mejor manera posible, convirtiéndose en una tarea de gran relevancia en las empresas, creando lo necesario para el éxito en su funcionamiento (Simon, 1998; Koontz, 2002). La TC menciona que la empresa debe estar encaminada a la toma de decisiones, tratando siempre de formular políticas, objetivos y principios que sean efectivos (Simon, 1998).

De acuerdo con Leuhmann (1997), para tomar una decisión racional es importante que primero se realice una comprensión de la empresa, y saber lo que sucede a gran detalle con ella y con la máxima autoridad, además de considerar hacer una reflexión de preparación que posea como propósito la buena toma de decisiones dentro de las organizaciones. Otro aspecto a considerar al tomar cualquier decisión es el trabajo en equipo, procurando que siempre esté enfocado a lograr un fin y encaminado a tener éxito (Chester, 1938).

Un aspecto critico a la TC, son las implicaciones que resultan de tomar una decisión, analizando los efectos que pueda traer consigo, ya que según Ibarra y Montaño (1992), la racionalidad controla a la organización, por ende, a los trabajadores queriendo asegurar que las decisiones tomadas sean efectivas, por lo que la TC no es más que un medio para lograr la dominación, explotación y poder sobre los individuos, a través de mecanismos como lo es, la toma de decisiones.

\section{Teoría de nuevas relaciones humanas}

Asimismo, aparece la Teoría de nuevas relaciones humanas (TNRH) con Abraham Maslow, analizando a las organizaciones y sus necesidades para el correcto funcionamiento, tomando en cuenta la motivación e interesándose por sus actitudes, cualidades, sentimientos y escuchando a los trabajadores para intercambiar experiencias que puedan ser beneficiosas tanto para la organización como para los mismos empleados y, de esta manera, lograr una mejor percepción del clima organizacional (Herzberg, Mausner y Snyderman, 1959).

En simultaneo, la TNRH se enfoca en las necesidades de los seres humanos, menciona ndo que se encuentran dentro de una jerarquía de predomino, dependiendo de su grado de importancia y tomando en cuenta que existen ciertos prerrequisitos que se deben considerar para lograr satisfacerlas, tales como la libertad de comunicación, expresión, igualdad, justicia, honestidad, equidad, entre otros que son vitales para las personas (Maslow, 1991). Por lo cual, dentro de esta teoría se puede observar un liderazgo que se preocupa por los demás tomando las decisiones con 
base a la satisfacción de necesidades y motivación de los mismos empleados.

Por el contrario, Elizalde, Martí y Martínez (2006) establecen una crítica en contra de Maslow ya que este no ofrece una descripción amplia sobre el estado de trascendencia en su jerarquía de necesidades, al pasar de un estado a otro, posiblemente porque pocas personas han llegado a la autorrealización, alcanzando la honradez, creatividad, capacidad para resolver problemas, entre otros.

\section{Teoría institucional}

De igual forma, se presenta la Teoría institucional (TI), la cual consiste en un método de perfeccionar los procesos organizacionales desarrollándose de tal manera que sea posible eliminar diversas formas organizativas que ya no se consideran adecuadas, adaptándose al medio en el que se encuentran, tanto interno como externo; es decir, al entorno que las rodea y así tener benefic ios que aporten un valor a las partes involucradas (Berrone y Gómez-Mejía, 2009); (DiMaggio y Powell, 1983).

La TI menciona que los procesos se perfeccionan con base en reglas y procedimientos, además de regulaciones, incentivos, políticas, entre otros aspectos, pues según Meyer y Rowan (1977) el proceso de institucionalización implica que se deben considerar dichos preceptos que ayuden a conseguir un estatus ante la sociedad, teniendo en cuenta la estructura organizacional de las empresas, la realidad en la que se encuentran y lo que está alrededor de la misma, con la finalidad de conseguir más productividad, eficacia, eficiencia, rentabilidad y permanencia dentro del mercado competitivo.

No obstante, la TI tiene ciertas complejidades, lo que se ha convertido en blanco de críticas para otros autores pues presenta algunas implicaciones sociales, y esto es debido a que las organizaciones son consideradas anarquías, debido a que no se acoplan con otras, tienen incluso pocas restricciones y a ocasiones objetivos inciertos que no pueden llegar a cumplirse, además de no contar con tecnología avanzada (DiMaggio y Powell, 1983). De acuerdo con Maguire y Hardy (2009), pueden existir aspectos externos que incidan en la desinstitucionalización, omitiendo las prácticas relacionadas y teniendo como resultado diversas problemáticas.

\section{Teoría de recursos y capacidades}

Posteriormente se encuentra la Teoría de recursos y capacidades (TRC), dentro de ésta se 
puede percatar la forma de liderar, pues es considerada una fuente de crecimiento y desarrollo dentro del mercado (Fierro y Mercado, 2012) debido a que, el humano es de los más valiosos capitales en las organizaciones, por lo que tener un alto desempeño implica una correcta gestión, un buen ambiente de trabajo, así como un buen liderazgo. La TRC se origina con base al autor Wernerfelt (1984) cuando establece que los recursos y los productos de una organización eran lo mismo, ya que ambos los posee la empresa y son complementarios uno del otro, además, representan tanto una oportunidad que puede utilizar la empresa a su favor como una debilidad que puede llegar a perjudicarlas.

La TRC surge y evoluciona desde las teorías más antiguas que han buscado entender a las organizaciones, originándose a partir de 1980 en el análisis de diversos fenómenos que podrían afectar el éxito de las mismas, tales como la cultura, los recursos en general, entre otros (Barney, Ketchen y Wright, 2011). En ese sentido, la TRC se ha considerado como un elemento necesario para la estrategia permitiendo conseguir el éxito organizacional (Gent y Andalaft, 2007).

Por el contrario, una crítica presentada en la TRC es el nivel de análisis que se presenta, ya que López y Sabater (2000) afirman que lo adecuado es considerar a la organización como un todo al momento de determinar cuáles son sus recursos considerados como valiosos, es por eso que la aseveración de determinarlos como único referente plantea problemas de inconsistencia, ya que su valor depende del entorno en el que se desenvuelve la empresa al ser éste el encargado de la valoración.

\section{Teoría de las relaciones interorganizacionales}

Se hace hincapié en la Teoría de las relaciones interorganizacionales (TRI), considerada una alternativa que proviene de la TRC, debido a que las empresas se juntan con la finalidad de compartir los recursos escasos que poseen y conseguir ciertos beneficios, como la creación de productos innovadores o sustitutos, volviéndose más competitivas dentro del mercado, tomando en cuenta las funciones y el rol que juega cada quien, conociendo sus respectivas habilidades, destrezas, aptitudes y valores para adquirir mayor eficiencia (Evan, 1967).

No obstante, la TRI enfrenta diversas complicaciones cuando hacen alianzas y relaciones, y son llamados problemas fronterizos donde es necesario prestar atención a la parte interna de la organización, tales como reglas, formalización y estructura, además de unir esfuerzos para afrontar discrepancias en la resolución de conflictos, pues lo primordial es el logro de los objetivos y la conservación de las diversas normas y/o políticas organizacionales (Provan, 1995). 


\section{Anarquías organizacionales}

En cuanto a las anarquías organizacionales, es otra de las teorías analizadas, figurando aquellas organizaciones que tienen diversos problemas que no toman en consideración el avance tecnológico, existe mala organización, los objetivos no son claros, además de otras problemáticas que las vulnera, ya que las decisiones tomadas dentro éstas son en modalidad espontánea, es decir, sin planificación alguna (Cohen, March y Olsen, 1972).

No obstante, cabe destacar que el anarquismo no está en contra de las organizaciones, sino que éste favorece el desarrollo de la misma, ya que permite la toma de decisiones con base en acuerdos que sean voluntarios para lograr armonía entre todos los involucrados, basándose en el comportamiento social (Wachhaus, 2011), por lo que en medio de las anarquías viene consigo el aspecto del liderazgo, ya que es fundamental ejercerlo con responsabilidad, debido a que este aspecto ayuda a equilibrar tendencias y resolver problemáticas (De Miguel, 2004).

\section{Teoría neoclásica}

Por otro lado, se analiza también la teoría neoclásica (TN), la cual surgió con la finalidad de poder reformar y modernizar a la teoría clásica de la administración, teniendo en cuenta los conceptos que fueron usados anteriormente por Taylor y Fayol, como eficiencia, jerarquía, racionalidad, principios, etc. (Drucker, 1955). Asimismo, mostrando su importancia se menciona también sus ventajas, pues proporciona buenos resultados a la empresa, muestra una estructura comprensible y simple haciendo mención de cada una de las responsabilidades que tiene cada trabajador, además de que promueve una idea de armonía entre pensadores e investigadores (Félix, 2001).

Sin embargo, la TN es criticada por diversos autores, ya que estimula la reducción del personal, la autoridad es única, debe respetarse y hacerse cargo de todas las actividades a la vez y no de una en específico, por lo que trae consecuencias al no centrarse solo en una actividad (Félix, 2001). Así pues, de acuerdo con Velázquez (2002), la TN está enmascarada por principios como la división del trabajo, autoridad y responsabilidad, además debe haber especialización de procesos, áreas y clientes.

\section{Teoría de gestión del conocimiento}

Posteriormente, aparece la Teoría de gestión del conocimiento (TGC) donde Nonaka y 
Takeuchi (1995) piensan que es necesario crear conocimiento nuevo a la empresa y poder difundirlo a través de todos los niveles jerárquicos e incorporarlo dentro de lo que ofrecen como productos y servicios, además de considerarse un aspecto clave para que empiecen a innovar y seguir hacia adelante, consiguiendo estabilidad y reducción de incertidumbre, una nueva estructura en la organización de mayor flexibilidad, adaptación, dinamismo y participación, todo ello, concentrando la energía y el esfuerzo grupal en lograr un objetivo en común y así lograr avances significativos que estén encaminados al desarrollo de la organización, además de poder definir los roles de cada empleado, empezando por colocar al gerente en el puesto directivo, con la finalidad de contribuir con el éxito organizacional; sin embargo, es importante mencionar que solo las grandes organizaciones se preocupan por crear conocimiento pero no saben cómo explotarlo.

\section{Tendencias administrativas}

Finalmente, se hace hincapié en las Tendencias administrativas (TA), éstas son conocidas por ser herramientas que permiten a las empresas valerse por sí mismas dentro del mercado con el objetivo de asegurar su existencia dentro del mismo (Sánchez, 2017). Es importante mencionar que las TA obligan a tomar diversas acciones tales como tomar decisiones, hacer capacitaciones, trabajar en equipo, mejoras en procesos, valores, actitudes, entre otros aspectos, con la finalidad de poder resolver problemas logrando los objetivos y metas propuestas (Cetin, Sossa y Pinto, 2016).

Dentro de las TA más comunes se encuentran la reingeniería de procesos, el outsourcing, el empoderamiento (empowerment), Benchmarking, sistema Kaizen y Coaching (Cetin, Sossa \& Pinto, 2016), donde todos tienen sus propios beneficios; sin embargo, también poseen implicacio ne s que generan ciertas desventajas por las cuales son consideradas puntos débiles para críticas, ya que el uso indebido de ellas ha conducido a algunas organizaciones al fracaso, llevándolas a la quiebra y desaparición.

Ahora bien, en la Tabla 1, de forma integrada y resumida se presenta una relación de las diversas teorías administrativas, mostrando la forma en que se gestiona la organización, vinculándolas con el tipo de liderazgo ejercido dentro de cada una de ellas con base en las aportaciones analizadas. Lo anterior, permite afirmar que el liderazgo ha estado presente en éstas por mucho tiempo, manifestándose de diferente forma y perspectiva, pudiendo detectar que a través de él se puede obtener más productividad, eficiencia, racionalidad, equilibrio, prosperidad, autoridad, dominación y poder. Además de buenas relaciones, comunicación, mejor ambiente de trabajo, buena toma de decisiones, satisfacción de necesidades, motivación, optimización de 
procesos, mejor desempeño, organización, estabilidad, orden, centralización, innovación, trabajo en equipo, logro de objetivos, así como democracia, justicia y felicidad que según Ibarra y Montaño (1992) pueden lograr cualquier empresa y penetran en la esencia del ser humano.

\section{Conclusiones}

Ahora bien, cabe señalar que las teorías anteriormente presentadas se consideran contribuciones relevantes para las organizaciones, por su forma de presentar los hechos y como lograron evidenciar sus resultados siendo más eficaces a través del tiempo y hasta en la actualidad, sin embargo, a pesar de tener aspectos negativos que las posiciona en el centro de críticas por otros autores, fueron y seguirán siendo de gran ayuda en el ámbito de la gestión organizacional, pues las empresas necesitan aprender a tomar riesgos y debe existir una persona quien esté al mando de ésta, buscando siempre cumplir con los objetivos propuestos, es por ello que el líder tiene un papel de suma importancia dentro de ellas, y su forma de tomar decisiones dependerá del estilo de liderazgo ejercido.

Por lo cual, Blake y Mouton (1980) afirman que el liderazgo es considerado una pieza clave que ayuda a lograr las metas propuestas por la organización, existiendo diversos tipos de ellos, entre los más comunes y usados dentro de las organizaciones, actualmente se encuentran cinco: autocrático, siendo aquel que solo se interesa por dar órdenes, interesado más por los resultados que por las personas; democrático, conocido como participativo y armónico en el que se toman en cuenta a las personas a la hora de tomar decisiones.

De igual forma, el liderazgo Laissez Faire (dejarlo ser), donde permite que los empleados trabajen por sí solos, fijándose metas propias y los medios necesarios para lograrlo; transformacional, aquel que fomenta un cambio en la organización, él se compromete y subordina las actividades satisfaciendo las necesidades de las personas; finalmente, el transaccional, donde se otorgan premios e incentivos para asegurar que las actividades se lleven a cabo, además el líder tiene derecho a castigar a quien no obedece.

Cada aspecto alcanzado gracias al liderazgo en sus diferentes estilos ejercidos es lo que se ha logrado conseguir con la práctica de cada una de las teorías organizacionales analizadas, pues actualmente muchas empresas retoman dichas teorías para lograr el éxito y la permanencia dentro del mercado, es por ello que, es importante mencionar que las teorías sobre las organizaciones son muchas y han sido desarrolladas debido a que ayudan en gran medida guiando en las acciones y funciones de éstas (Hatch, 1997).

Así pues, el liderazgo ha sido y seguirá siendo parte fundamental dentro de las 
organizaciones y de la vida misma, considerándose relevante para su gestión y herramienta clave por varios autores, es por ello que, al ser esencial en el estudio de éstas, se ha buscado indagarlo detenidamente bajo este análisis histórico de las teorías organizacionales, demostrando así, que dicho término aparece de distintas formas pero con la finalidad de lograr los objetivos de cualquier organización y seguir subsistiendo a través del tiempo, conduciendo y guiándolas a obtener un alto rendimiento.

Finalmente, se puede decir que las organizaciones deben estar conscientes del estilo de liderazgo que ejerzan dentro de su gestión, debido a que puede traer consigo aspectos negativos como mala comunicación, bajo desempeño, escasa productividad, entre otros, que llevarán al fracaso si no se ejerce como tal, por lo que deben buscar la permanencia y prosperidad, por lo tanto, se recomienda para investigaciones futuras seguir analizando el aspecto de liderazgo, no solo al interior de las empresas, sino dentro de más ámbitos, tal como las clases sociales, religión, familia, entre otros, que permita tener un conocimiento de mayor amplitud sobre el abordaje de dicho elemento clave $\mathrm{y}$ fundamental que ha logrado ser relevante en el funcionamiento de las organizaciones.

\section{Referencias}

Arévalo, D. (2011). Relaciones de poder-saber en la doctrina administrativa de Henri Fayol. Ensayos de Economía. 21(39), 145-167. Recuperado de https://re vistas.unal.edu.co/index.php/ede/article/view/28644

Assalone, E. (2012). La caracterización de la burocracia en los principios de la filosofía del derecho de G.W.F. Hegel. Los inicios de la reflexión filosófica sobre la administración pública. Revista de Humanidades, 25, 29-44. Recuperado de https://www.redalyc.org/pdf/3212/321227326002.pdf

Barney, J. B., Ketchen, D. J., y Wright, M. (2011). The Future of resource-based theory. Journal of Management, 37(5), 1299-1315.

Berrone, P., \& Gómez-Mejia, L. R. (2009). Enviromental perfomance and executive compensation: an integrated agency-institutional perspective. Academy of Management Journal, 52(1), 103-126. Recuperado https://www.researchgate.net/publication/278305114_Environmental_Performance_and_E xecutive_Compensation_An_Integrated_Agency-Institutional_Perspective

Blake, R., y Mouton, J. (1980). El nuevo Grid gerencial. México: Editorial Diana.

Carreño, E. (2016). Burocracia y política exterior: los nuevos desafíos de la práctica diplomática. Revista del CLAD Reforma y Democracia, 65, 103-128. Recuperado de 
http $/ /$ old.clad.org/portal/publicaciones-del-clad/revista-clad-reformademocracia/articulos/065-junio-2016/Carreno.pdf

Carrión, N. (2017). Modelo de liderazgo basado en el coaching para el directivo de una empresa hotelera en la ciudad de Piura (Trabajo de Suficiencia Profesional de licenciatura en Administración de Empresas). Universidad de Piura. Facultad de Ciencias Económicas y Empresariales. Programa Académico de Administración de Empresas. Piura, Perú. Recuperado de https://pirhua.udep.edu.pe/handle/11042/2869

Cetin, O., Sossa, C., \& Pinto, B. (2016). Tendencias administrativas: el empoderamiento en las empresas. Econógrafos, 120-140. Recuperado de https://www.academia.edu/26351597/TENDENCIAS_ADMINISTRATIVAS_EL_EMPO DERAMIENTO_EN_LAS_EMPRESAS

Chester, B. (1938). The functions of the executive. Estados Unidos: Harvard University Press. Cohen, M., March., \& Olsen, J. (1972). El bote de basura como modelo de elección organizacional. Administrative Science Quarterly, 17(1), 1-25. Recuperado de $\mathrm{http} / / \mathrm{www}$.scielo.org.mx/pdf/gpp/v20n2/v20n2a2.pdf

Crozier, M. (1974). Elfenómeno burocrático. Buenos Aires: Editoriales Amorrortu.

Da Silva, R. (2007). Escuela de relaciones humanas y su aplicación en una empresa de telecomunicaciones. Scientia et Technica, XIII(34), 309-314. Recuperado de https://www.redalyc.org/pdf/849/84934052.pdf

De Miguel, J. M. (2004). Las universidades como anarquías organizadas Reis. Revista Española de Investigaciones Sociológicas, 106, 177-220. Recuperado de https://www.redalyc.org/pdf/997/99717667007.pdf

DiMaggio, P., \& Powell, W. (1983). The iron cage revisited: Institutional isomorphism and collective rationality in organizational fields. American Sociological Review, 48(2), $147-$ 160. Recuperado de https://www.uio.no/stud ier/emner/matnat/ifi/INF9200/v10/readings/papers/DeMaggio.pdf

Drucker, P. F. (1955). "Management science" and the manager. Management Science, 1(2), 115-126.

Elizalde, H., Martí, M., y Martínez, F. (2006). Una revisión crítica del debate sobre las necesidades humanas desde el enfoque centrado en la persona. Revista de la Universidad Bolivariana, 5(15), 1-18. Recuperado de https://www.redalyc.org/articulo.oa?id=30517306006

Estrada, S. (2007a). Liderazgo a través de la historia. Scientia et Technica, XIII(34), 343-348. Recuperado de https://www.redalyc.org/pdf/849/84934058.pdf

Estrada, S. (2007b). Predominio del estilo de liderazgo en la evolución de la administración. Scientia et Technica, XIII(35), 287-292. Recuperado de https://revistas.utp.edu.co/index.php/revistaciencia/article/view/5445 
Evan, W. (1967). La órbita de la organización: Hacia una teoría de las relaciones Interorganizacionales. Buenos Aires: OMEBA.

Fayol, H. (1987). Administración industrial y general. México: Suc. Herrero Hnos.

Félix, J. (2001). ¿Por qué el predominio de la teoría neoclásica? Cuad. Econ, 20(34), 81-291. Recuperado de http://www.scielo.org.co/scielo.php?script=sci_arttext\&pid=S0121 47722001000100015

Fierro, M., y Mercado, S. (2012). La innovación organizativa y sus predictores desde la teoría de los recursos y capacidades. Estudios Gerenciales, 28, 109-123. Recuperado de http//www.scielo.org.co/pdf/eg/v28nspe/v28nspea07.pdf

Fink, A. (2010). Conducting research literature review: from the internet to paper. Estados Unid os: SAGE Thousand Oaks.

García, J., y Dutchke, G. (2007). Las organizaciones con capacidad de aprendizaje. A propósito de una revisión literaria. Acimed, 16(5), 1-22. Recuperado de http $/ /$ scielo.sld.cu/scielo.php?script=sci_arttext\&pid=S1024-94352007001100005

Gent, K., y Andalaft, A. (2007). Extensión de los postulados de la teoría de los recursos y las capacidades de la firma a campos psicológicos. Revista Economía y Administración, 68, 35-

56. Recuperado de http//www2.udec.cl/ rea/REVISTA\%20PDF/Rev68/rea68art3.pdf

Gil, F., Alcover, C., Rico, R., y Sánchez, M. (2011). Nuevas formas de liderazgo en equipos de trabajo. Papeles Del Psicólogo, 32(1), 38-47. Recuperado de https://www.redalyc.org/pdf/778/77817210005.pdf

Gillespie, R. (1991) Manufacturing knowledge: A history of the Hawthorne experiments. Estados Unidos: Cambridge University Press.

Hatch, M. (1997). Organization theory. Modern, symbolic and postmodern perspectives. Estados Unidos: Oxford University Press.

Herzberg, F., Mausner, B., y Snyderman, B. (1959). Motivation at work. Inglaterra: Transaction publishers

Ibarra, E., y Montaño, L. (1992). Mito y poder en las organizaciones. Un análisis crítico de la teoría de la organización. México: Trillas

Koontz, H. (2000). Revisión de la jungla de la teoría administrativa. Revista Contaduría y Administración, 199, 55-74. $\quad$ Recuperado de http //www.ejournal. unam.mx/rca/199/RCA19903.pdf

Leuhmann, N.(1997). Organización y decisión. Autopoiesis, acción y entendimiento comunicativo. Universidad Iberoamericana. México: Anthropos

López, J., y Sabater, R. (2000). La teoría de los recursos y capacidades de la empresa. Una revisión. Recuperado de https://www.academia.edu/30047380/la_teor\%c3\%8da_de_los_recursos_y_capacidades_de 
_la_empresa._una_revisi\%c3\%93n

Maguire, S., y Hardy, C. (2009). Discourse and deinstitutionalization: The Decline of DDT. Academy of Management Journal, 52(1), 148-178. Recuperado de https://journals.aom.org/doi/10.5465/amj.2009.36461993

Maslow, A. (1991). Teoría de la motivación Humana. España: Ediciones Días de Santos

Mayo, E. (1946). Problemas humanos de una civilización industrial. Argentina: Ediciones Nueva Vision, S.A.I.C.

Meyer, J. W., y Rowan, B. (1977). Institutionalized organizations: Formal structure as myth and ceremony. American Journal of Sociology, 83(2), 340-363.

Mouzelis, N.(1975). Organización y burocracia. Barcelona: Ediciones Península.

Nonaka, I. (2007). La empresa creadora de conocimiento. Harvard Business Review, 1-10.

Palomo, M. (2013). Liderazgo y motivación de equipos de trabajo. Madrid: ESCI Editorial

Posner, B., y Kouzes, J. (2011). Leadership is a relationship. Wiley Online Library, 1-21. https://doi.org/10.1002/9781118983867.ch1

Preciado, A., y Gómez, M. (2008). El liderazgo en la gestión de la empresa informativa. Revista Ciencias Estratégicas, 16(19), 79-96. Recuperado de https://www.redalyc.org/pdf/1513/Resumenes/Resumen_151312831006_1.pdf

Prentice, W. (2004). Comprendiendo el liderazgo. Harvard Business School Publishing Corporation, 2-18.

Provan, K. G. (1995). A preliminary theory of interorganizational network effectiveness: A connparative study of four connmunity mental health systems. Administrative Science Quarteriy, 40, 1-33.

Sánchez, J. (2017). Las principales tendencias de la administración moderna. Linkedin. Recuperado de https://www.linkedin.com/pulse/las-8-principales-tendencias-de-laadministraci\%C3\%B3n-s\%C3\%A1 nchez-morz\%C3\%A1 n

Simon, H. (1988). El comportamiento administrativo. Estudio de los proceso decisorios en la organización administrativa. Argentina: Aguilar S. A Ediciones

Taylor, F. W. (1983). Principios de la administración científica. México: Herrero Hermanos.

Velázquez, F. (2002). Escuelas e interpretaciones del pensamiento administrativo. Estudios gerenciales, 18(83), 31-55. Recuperado de https://www.redalyc.org/pdf/212/21208302.pdf

Wachhaus, T. (2011). La anarquía como modelo para la gobernanza de la red. The American Society for Public Administration, 72(1), 33-42.

Weber, M. (1973). ¿Qué es la burocracia? México: Ediciones Coyoacán

Weber, M. (1992). Economía y sociedad. México: Fondo de cultura económica 
Wernerfelt, B. (1984). A resource-based view of the firm. Strategic management Journal, 5: 171180.

Yukl, G. (2003). Leadership in organizations. New York: Pearson. 


\section{Anexos}

Tabla 1.

Liderazgo a través de la teoría de la organización

\begin{tabular}{c}
\hline \multicolumn{1}{c}{ Teoría } \\
\hline $\begin{array}{l}\text { Administración } \\
\text { científica }\end{array}$
\end{tabular}

Administración industrial

Henry Fayol (1987)

Relaciones
humanas

Burocracia

Max Weber

(1973)
Teoría del

Comportamiento
Herberth Simon (1988)
Forma de liderar

\section{Tipo de \\ liderazgo \\ en la actualidad}

Autocrático

El liderazgo está basado por medio del control formal a través de la súper especialización con supervisores por actividad y departamento pensante, no permitiendo que ellos participen en la toma decisiones.

Se establecía dentro de los principios una unidad de mando y la autoridad para dar órdenes a través de niveles jerárquicos definidos con remuneración justa para asegurar las actividades se lleven a cabo.

El liderazgo estaba basado por medio del control en los grupos sociales dentro de la organización, preocupándose por la motivación y un ambiente de trabajo armónico.

La base principal de la burocracia era el poder, un poder legítimo a través de normas y reglamentos para asegurar la seguridad laboral, dominación y autoridad.

Transaccional

Democrático

Autocrático

Democrático

A través de un liderazgo adecuado que fuese de ayuda para una buena toma de decisiones racional.

Abraham Maslow Mediante la motivación y un buen liderazgo.

Democrático (1991)

Di Maggio y Las órdenes son dadas por los altos mandos asegurándose de que se lleven a cabo Autocrático
Powell (1983),

Meyer y Pwan

(1977), Scott

(1977) mediante la institucionalización, un proceso donde los empleados aceptan ciertas reglas o pautas a lo largo del tiempo. 


\section{Teoría de las relaciones interorganizacio nales}

\section{Anarquías organizacionales}

Teoría

Neoclásica de la

Administración

Teoría basada en

la Gestión del

Conocimiento

Tendencias Administrativas
Evan (1967) y

Provan (1995)

Las importante para potencializar recursos, fomentando el cambio así como el desempeño de la organización, coordinándolas y

liderándolas correctamente.

Cohen, March \& Olsen, 1972

Peter Druker (1995)

Nonaka y Takeuchi (1995

El liderazgo forma parte de una buena toma decisiones mediante acuerdos acoplando los sistemas permite conseguir armonía, paz, estabilidad y orden.

La centralización, jerarquía, división del trabajo se hacen presentes en la forma de liderar delegando y

concentrando la autoridad y responsabilidad.

Se basa en un modelo de proceso de gestión llamado de media arriba abajo donde el gerente es el que tiene el rol de mandar y crear conocimiento, es además donde se concentra la autoridad tomando en cuenta a todos los niveles jerárquicos.

Cetin, O., Sossa, El enfoque de liderazgo es cambiado, desde C., \& Pinto, B. (2016). uno piramidal a un liderazgo armónico y participativo, donde se promueve el cambio y el compromiso.

\section{Transformacio}

nal

Laissez faire

Autocrático

Democrático

Democrático y Transformacio

nal

Nota: Elaboración propia con base en los autores antes mencionados 